EESTI NSV TEADUSTE AKADEEMIA TOIMETISED. IX KÖIDE

FOOSIKALIS-MATEMAATILISTE JA TEHNILISTE TEADUSTE SEERIA. 1960, NR. 4

ИЗВЕСТИЯ АКАДЕМИИ НАУК ЭСТОНСКОИ ССР. ТОМ IX СЕРИЯ ФИЗИКО-МАТЕМАТИЧЕСКИХ И ТЕХНИЧЕСКИХ НАУК. 1960, № 4

\title{
К ТЕОРИИ ЭЛЕКТРОННО-КОЛЕБАТЕЛЬНЫХ СПЕКТРОВ МОЛЕКУЛ И КРИСТАЛЛОВ
}

\author{
К. РЕБАНЕ,
}

кандидат физико-математических наук

\section{о. сильд}

В работе дан простой вывод формулы для моментов спектральной полосы, возникающей в результате электронно-колебательного перехода в кристалле или в молекуле. Результаты совпадают с формулами Лэкса ['], но наш вывод проще. Ясно видно, что нет необходимости в описании колебательного движения ядер нормальными колебаниями, а распределение систем по колебательным уровням не обязательно равновесно. Полосы могут иметь также частично или полностью линейчатую структуру. Кроме того, основная формула для моментов справедлива и вне адиабатического прнближения.

Применение полученных формул иллюстрируется рассмотрением связи между контурами полос поглощения и излучения. В частности, получены достаточные и необходимые условия появления зеркальной симметрни полос (Левшин [2]) и найдена простая связь между полосами для случая, когда в электронном переходе изменяются и силовые константы колебаний.

В теории электронно-колебательных переходов в сложных колебательных системах (кристаллы, сложные молекулы) в последние годы стали широко применяться различные однокоординатные методы рассмотрения $\left[{ }^{3-10}\right]$. При всех успехах применения различных однокоординатных моделей к сложным колебательным системам и совершенно независимо от того, в какой мере в будущем удастся доказать справедливость этих моделей при рассмотрении тех или иных конкретных явлений, не следует забывать о заведомо грубой природе однокоординатных моделей. При появлении определенных расхождений между экспериментом и теорией, базирующейся на методе одной конфигурационной координаты, всегда заново возникает основной вопрос: не обусловлено ли расхождение слишком грубым описанием колебательного движения. Строго говоря, лишь после того, как убедительно показано, что то или иное расхождение нельзя объяснить действием множества колебаний, не включенных в модель, следует искать другие причины расхождения. Ясно, что далеко не всегда требуемый анализ возможен, и вопрос о роли всей совокупности колебаний остается зачастую открытым. Применяя при рассмотрении электронно-колебательных процессов однокоординатную модель, чаще всего приходится пренебрегать огромным количеством колебаний не потому, что это теоретически оправдано, а просто потому, что мы еще не умеем учитывать большое число колебаний.

3 ENSV TA Toimetised T-4 60. 
Рассмотрение колебательного движения сложной системы как совокупности квазинезависимых нормальных колебаний является, безусловно, гораздо более точным решением проблемы. В настоящее время существует ряд работ, где на основе различных вариантов этой модели удалось последовательно провести рассмотрение электронно-колебательных переходов с учетом многих колебательных степеней свободы (см., например, $[1,11,12])$.

Рассмотрение этих моделей приводит к несколько более сложным вычислениям. При этом теряется также исключительная простота и наглядность однокоординатной модели. Поэтому методы и результаты этих работ не пользуются, к сожалению, такой популярностью среди экспериментаторов, как, например, метод потенциальных кривых.

Однако, даже законность введения квазинезависимых нормальных колебаний иногда вызывает сомнения. Так, Степанов [9] пришел к выводу, что в случае сложной молекулы вообще нельзя вводить квазинезависимые нормальные колебания.

В центрах люминесценции в кристаллофосфорах, особенно в ионных кристаллах, стоксовы потери весьма значительны. Поэтому следует ожидать, что непосредственно госле перехода, в результате которого изменилось электронное состояние центра, а также на первых этапах процесса релаксации избыточной колебательной энергии амплитуды смещений ближайших к активатору ионов велики. Тогда велико и влияние ангармоничности колебаний и представление о квазинезависимых нормальных колебаниях может быть весьма грубым.

Поэтому любопытно получить некоторые результаты, не зависящие от того, много ли колебательных степеней свободы у системы или мало, применимо ли представление о квазинезависимых нормальных колебаниях или нет.

Ниже на примере исследования связи между контурами полос поглощения и излучения показано, как некоторые подобные результаты могут быть получены. Целесообразным подходом к решению нашей задачи является описание спектральных полос методом моментов, предложенное Лэксом [1].

\section{1. Формулы для моментов спектральных полос}

Спектры поглощения и излучения в кристаллофосфорах часто имеют характерный колоколообразный контур. Этот контур хорошо описывается гауссовой кривой, умноженной (в целях учета небольшой асимметрии полосы) на некоторую «весьма гладкуюх поправочную функцию. Аналогичный контур имеют многие функции распределения, встречающиеся в теории вероятностей. Для количественной характеристики таких функций распределения в теории вероятностей применяется хорошо известный метод моментов (см., например, $\left.\left[{ }^{13}\right]\right)$.

Момент $S_{l}$ порядка $l$ функции распределения $\varrho(x)$ определяется интегралом

$$
S_{l}=\int x^{l} \varrho(x) d x,
$$

где интегрирование проводится по всей области изменения $x$. Формула (1) дает моменты, взятые относительно начала координат. Такие моменты будем называть, так же как в теории вероятностей, н а ч а ль ны м и моментами. Если переменная является дискретной величиной, интеграл заменяется соответствующей суммой

$$
s_{l}=\sum_{s} x_{s}^{l} \varrho_{s}
$$

Интеграл Стилтьеса

$$
S_{l}=\int x^{l} d F(x)
$$

где $F(x)$ - интегральная функция распределения, объединяет в себе обе формулы 
(1) и (2). Если $F(x)$ имеет производную и является интегралом от нее, то $d F(x)=$ $=\varrho(x) d x$ и мы получаем (1). Если изменения $F(x)$ происходят только в точках $x_{1}$, $x_{2}, \ldots$, то $S_{l}$ сводится к сумме и мы приходим к (2). Но особых преимуществ по существу запись с помощью интеграла Стилтьеса здесь не дает, и в данной статье мы не будем ею пользоваться.

Если для наглядности толковать $\varrho(x)$ как распределение массы некоторого тела вдоль оси $x$, то $S_{0}$ выражает массу тела. Первый момент связан с координатой центра тяжести $\bar{x}$ тела формулой

$$
\bar{x}=\frac{S_{1}}{S_{0}} .
$$

Второй момент $S_{2}$ связан с моментом инерции.

Если $Q(x)$ колоколообразная кривая, то $\bar{x}$ близка к координате максимума $Q(x)$, а

$$
\bar{S}_{2} \equiv S_{2}-\frac{S_{1}^{2}}{S_{0}}=\overline{x^{2}}-S_{0}(\bar{x})^{2},
$$

т. е. второй момент, взятый относительно $\bar{x}$ («момент инерции» тела относительно его «центра тяжести»), характеризует ширину распределения. (Моменты, взятые относительно $\bar{x}$, называются центральными и обозначаются чертой над символом момента.) Аналогично $\overline{S_{3}}$ характеризует уже асимметрию ${ }^{*}(x)$ и т. д.

Для описания спектральной полосы в кристаллофосфорах обычно дают положение ее максимума и полуширину. Иногда вводится еще параметр, характеризующий асимметрию (см., например, $\left[{ }^{6},{ }^{14}\right]$ ).

Благодаря близости контура полосы к гауссовой кривой, несколько ее первых моментов дают столько же информации о полосе, сколько применяемые обычно положение максимума, полуширина и т. д. Определение моментов для экспериментально полученной кривой несколько более затруднительно (и, вероятно, просто несколько непривычно), тем определение используемых обычно параметров, но никаких трудностей по существу здесь не возникает. Вместе с тем теоретическое вычисление моментов спектральной кривой значительно проще, чем вычисление самой кривой или ее обычно используемых характеристик. Именно для моментов спектральных кривых, как это впервые показал Лэкс ['], можно получить ряд общих результатов.

Ниже будет дан весьма простой, как нам кажется, вывод формулы для моментов спектральной полосы, возникшей в результате электронноколебательного перехода. Рассмотрение относится в равной мере к кристаллам и к молекулам, как к сложным, так и к двухатомным. Наши результаты совпадут с формулами Лэкса [1], но при нашем выводе особенно хорошо видно, что эти формулы имеют широкие границы применимости. В частности, колебательное движение ядер может и не описываться нормальными колебаниями, а распределение систем по колебательным уровням не обязательно равновесно. Полосы могут иметь также частично или полностью линейчатую структуру.

Момент порядка $l$ спектральной кривой $S_{l}$ определяется выражением

$$
S_{l}=\sum_{i} n_{i} \sum_{s}\left(E_{s}-E_{i}\right)^{l} W_{s i}
$$

$$
\text { Здесь фактор Франка-Кондона } \quad W_{s i}=\left[\int \psi_{I I s}^{*}(x) M(x) \psi_{I i}(x) d x\right]^{2} \text {; }
$$

- Для характеристики полосы можно пользоваться еще т. н. абсолютными центральными моментами, в частности первым абсолютным центральным моментом, т. е. величиной $\bar{S}_{1}^{a}=\int|x-\bar{x}| \varrho(x) d x$. 
$n_{i}$ - число систем на исходном колебательном уровне номера $i$; $E_{i}$ и $E_{s}$ - соответственно энергии исходного и конечного колебательного уровней; $M(x)$ - электронный матричный элемент. $\psi_{\mathrm{I} i}(x)$ и $\psi_{\mathrm{II} s}(x)$ являются колебательными волновыми функциями в электронных состояниях I (исходное) и II (конечное); $x$ означает набор всех колебательных координат ядер, причем не предполагается разделение колебательного движения на отдельные нормальные координаты. $i$ и $s$ являются соответствующими наборами колебательных квантовых чисел. Во избежание обилия индексов будем опускать индексы электронного состояния всюду, где это не вызывает недоразумений.

Мы пишем формулы для дискретных колебательных уровней, но все дальнейшее справедливо также в случае сплошного спектра. Кроме того, из (5) видно, что мы говорим о распределениях интенсивности, которые получаются, если измеренные в энергетических величинах и нормированные экспериментальные контуры полос умножены соответственно на $v^{-4}$ (излучение) и $v^{-1}$ (поглощение). Подробнее об этом см., например, $\left[{ }^{15}\right]$.

Рассмотрим величину

$$
\begin{gathered}
\left(E_{s}-E_{i}\right)^{t} \psi_{\mathrm{IIs}}^{*}(x) M(x) \psi_{\mathrm{I} i}(x)=\sum_{k=0}^{l}(-1)^{k} C_{l}^{k} E_{s}^{l-k} \psi_{\mathrm{IIs}}^{*}(x) M(x) E_{i}^{k} \psi_{\mathrm{I} i}(x)= \\
=\sum_{k=0}^{l}(-1)^{k} C_{l}^{k} M(x)\left[\widehat{H}_{\mathrm{I}}^{k} \psi_{\mathrm{I} i}(x)\right]\left[\widehat{H}_{\mathrm{II}}^{l-k} \psi_{\mathrm{II} s}(x)\right]^{*} .
\end{gathered}
$$

Здесь $\widehat{H}_{\mathrm{I}}$ и $\widehat{H}_{\mathrm{II}}$ - операторы Гамильтона, описывающие колебания ядер в адиабатическом приближении.

Проинтегрируем полученное равенство по $x$ и, используя свойство самосопряженности операторов $\widehat{H}_{\mathrm{II}}^{l-k}$, преобразуем интегралы. Тогда

$$
\begin{gathered}
\left(E_{s}-E_{i}\right)^{l} \int \psi_{s}^{*}(x) M(x) \psi_{i}(x) d x= \\
=\sum_{k=0}^{l}(-1)^{k} C_{l}^{k} \int \psi_{s}^{*}(x) \widehat{H}_{\mathrm{II}}^{l-k} M(x) \widehat{H}_{\mathrm{I}}^{k} \psi_{\mathrm{i}}(x) d x .
\end{gathered}
$$

Умножим (7) на интеграл $\int \psi_{i}^{*}\left(x^{\prime}\right) M^{*}\left(x^{\prime}\right) \psi_{s}\left(x^{\prime}\right) d x^{\prime}$, проведем суммирование полученных из (7) уравнений по всем конечным колебательным состояниям $s$ и используем условие полноты $\sum_{s} \psi_{s}^{*}(x) \psi_{s}\left(x^{\prime}\right)=$ $=\delta\left(x-x^{\prime}\right)$. Получаем

$$
\sum_{s}\left(E_{s}-E_{i}\right)^{l} W_{s i}=\sum_{k=0}^{l}(-1)^{k} C_{l}^{k} \int \psi_{i}^{*}(x) M^{*}(x) \widehat{H}_{\mathrm{II}}^{l-k} M(x) \widehat{H}_{I}^{k} \psi_{i}(x) d x .
$$

Для получения момента порядка $l$ проведем еще усреднение по начальным колебательным уровням и окончательно имеем

$$
S_{l}=\sum_{i} n_{i} \sum_{k=0}^{l}(-1)^{k} C_{l}^{k} \int d x\left\{\psi_{i}^{*}(x) M^{*}(x) \widehat{H}_{\mathrm{I}}^{l-k} M(x) \widehat{H}_{\mathrm{I}}^{k} \psi_{i}(x)\right\} .
$$

Для нулевого момента получаем отсюда

$$
S_{0}=\sum_{i} n_{i} \int|M(x)|^{2}\left|\psi_{i}(x)\right|^{2} d x
$$


или в приближении Кондона *

$$
S_{0} \cong|M|^{2} N,
$$

где $N$ - число систем в исходном электронном состоянии. Следующие три момента:

$$
\begin{gathered}
S_{1}=\sum_{i} n_{i} \int d x\left\{\psi _ { i } ^ { * } ( x ) \left[M^{*}(x) \widehat{\left.\left.H_{\mathrm{II}} M(x)-|M(x)|^{2} \widehat{H}_{\mathrm{I}}\right] \psi_{i}(x)\right\} \cong}\right.\right. \\
\cong|M|^{2} \sum_{i} n_{i} \int d x\left\{\psi_{i}^{*}(x)\left[\widehat{H_{\mathrm{II}}}-\widehat{H_{\mathrm{I}}}\right] \psi_{i}(x)\right\}=|M|^{2} \int U(x) \mathrm{Q}(x) d x,
\end{gathered}
$$

где $\mathrm{Q}(x) \equiv \sum_{i} n_{i} \mid \psi_{i}(x)^{2}$ является функцией распределения координат и $U(x)=U_{\text {II }}(x)-U_{I}(x)$ представляет собой разность между адиабатическими потенциалами конечного и исходного электронных состояний.

$$
\begin{gathered}
S_{2}=\sum_{i} n_{i} \int d x\left\{\psi _ { i } ^ { * } ( x ) \left[M^{*}(x) \widehat{H}_{\mathrm{II}}^{2} M(x)-2 M^{*}(x) \widehat{H}_{\mathrm{II}} M(x) \widehat{H}_{\mathrm{I}}+\right.\right. \\
\left.\left.+|M(x)|^{2} \widehat{H}_{\mathrm{I}}^{2}\right] \psi_{i}(x)\right\} \cong|M|^{2} \sum_{i} n_{i} \int d x\left\{\psi_{i}^{*}(x)\left[\widehat{H}_{\mathrm{II}}^{2}-2 \widehat{H}_{\mathrm{II}} \widehat{H_{\mathrm{I}}}+\widehat{H}_{\mathrm{I}}^{2}\right] \psi_{i}(x)\right\}= \\
=|M|^{2} \int U^{2}(x) \varrho(x) d x . \\
S_{3}=\sum_{i} n_{i} \int d x\left\{\psi _ { i } ^ { * } ( x ) \left[M^{*}(x) \widehat{H}_{\mathrm{II}}^{3} M(x)-3 M^{*}(x) \widehat{H}_{\mathrm{II}}^{2} M(x) \widehat{H}_{\mathrm{I}}+\right.\right. \\
\left.\left.\quad+3 M^{*}(x) \widehat{H}_{\mathrm{II}} M(x) \widehat{H}_{\mathrm{I}}^{2}-|M(x)|{ }^{2} \widehat{H}_{\mathrm{I}}^{3}\right] \psi_{i}(x)\right\} \cong \\
\cong|M|^{2} \sum_{i} n_{i} \int d x\left\{\psi_{i}^{*}(x)\left[\widehat{H}_{\mathrm{II}}^{3}-3 \widehat{H}_{\mathrm{II}}^{2} \widehat{H}_{\mathrm{I}}+3 \widehat{H}_{\mathrm{II}} \widehat{H}_{\mathrm{I}}^{2}-\widehat{H}_{\mathrm{I}}^{3}\right] \psi_{i}(x)\right\}
\end{gathered}
$$

Центральные моменты $\bar{S}_{l}$ (моменты, взятые относительно средней энергии $\bar{E}$ полосы) определяются формулой

$$
\bar{S}_{l}=\sum_{i} n_{i} \sum_{s}\left(E_{s}-E_{i}-\bar{E}\right)^{l} W_{s i},
$$

где $\bar{E}=S_{1} / S_{0}$.

$\bar{S}_{l}$ выражается через $S_{l}$ формулой

$$
\overline{S_{l}}=\sum_{k=0}^{l}(-1)^{k} C_{l}^{k}\left(\frac{S_{1}}{S_{0}}\right)^{k} S_{l-k}
$$

Для нескольких первых моментов имеем отсюда

$$
\begin{aligned}
& \left.\bar{S}_{0}=S_{0} \quad \text { (площадь под спектральной кривой }\right) \\
& \bar{S}_{1}=0 \\
& \bar{S}_{2}=S_{2}-\frac{S_{1}^{2}}{S_{0}}=S_{0}\left[\overline{\mathrm{E}^{2}}-(\overline{\mathrm{E}})^{2}\right]
\end{aligned}
$$

- Прнближение Кондона означает ограничение в разложении $M(x)$ по степеням $x$ постоянным членом. 


$$
\bar{S}_{3}=S_{3}-3 S_{2} \frac{S_{1}}{S_{0}}+2 \frac{S_{1}^{3}}{S_{0}^{2}} .
$$

Отметим еще, что формула (8) справедлива не только в адиабатическом приближении: при ее выводе использовалось лишь свойство полноты системы функции $\psi_{s}$ и предполагалось, что $M(x)$ является оператором умножения.

Например, из формулы (8) для $S_{1}$ можно весьма просто получить доказательство известной теоремы о сумме сил осцилляторов для водородоподобного атома [1б. Для э1ого следует лишь трактовать $M(x)$ как проекцию радиус-вектора электрона на направление электрического вектора световой волны и принять $\widehat{H}_{\mathrm{II}}=\widehat{H}_{\mathrm{I}}=\widehat{H}$, где $\widehat{H}$ оператор Гамильтона для водородоподобного атома. Из формулы (8) нами получены также некоторые обобщения упомянутой теоремы, которые будут изложены в отдельной заметке.

Для полосы излучения начальные моменты нечетного порядка, определяемые по формуле (5), отрицательны. Это обусловлено просто тем, что при применении для полосы излучения формул, записанных для поглощения, мы имеем $E_{s}-E_{i}<0$. Обычно энергия излучения рассматривается как положительная величина. Поэтому мы в дальнейшем будем считать, что в случае излучения у нас в (5) фигурирует разность $E_{i}-E_{s}$ и что все нечетные начальные моменты также положительны.

\section{2. Условия появления зеркальной симметрии полос поглощения и излучения}

Обнаруженная Левшиным зеркальная симметрия полос поглощения и излучения, соответствующих электронно-колебательным переходам между двумя электронными состояниями, является одним из наиболее ярких экспериментальных фактов о спектрах сложных люминесцирующих молекул *. Условия появления зеркальной симметрии, которым должны удовлетворять системы электронно-колебательных уровней и вероятности переходов, были выяснены уже самим Левшиным [2] (см. формулы (14)). Пекар [1'] при помощи квантовомеханического расчета показал, что спектральные полосы системы, колебания которой описываются в гармоническом приближении и частоты колебаний не изменяются в результате электронного перехода, обладают зеркальной симметрией.

Однако, по существу оставался без ответа вопрос о том, не может ли появиться зеркальная симметрия еще при каких-нибудь условиях, отличных от условий Левшина. Этот вопрос кажется особенно законным, если иметь в виду, что речь идет о сложных молекулах, где важное место должны занимать средние статистические свойства молекулы и даже существенные различия в деталях не обязательно ярко проявляются. Имеются работы $\left[{ }^{18,}{ }^{19}\right]$, авторы которых пришли к заключению, что зеркальная симметрия не обязательно связана с выполнением условий Левшина, а возможна как результат лишь некоторой «статистической равноценности» состояний сложной молекулы.

Ниже мы сформулируем условия Левшина непосредственно для адиабатических потенциалов, т. е. в виде, свободном от необходимости

* Связь между контурами полос в другом аспекте (связь $x(v)$ и $l(v)$ при о д н й и той же частоте v) установлена Степановым [17]. Его формулы справедливы при весьма общих условиях и исключительно полезны в тех случаях, когда полосы поглощения и излучения в значительной степени взаимно перекрываются. 
рассмотрения нормальных колебаний, и покажем, что упомянутые условия являются не только достаточным и, а практически также н еоб ходи мы ми.

Условие зеркальной симметрии можно записать через моменты спектральных кривых следующим образом:

$$
S_{l}^{t}\left(E_{0}\right)=(-1)^{l} S_{l}^{l}\left(E_{0}\right),
$$

где $S_{l}{ }^{x}\left(E_{0}\right)$ - момент полосы поглощения порядка $l$ относительно «точки симметрии» $E_{0} ; S_{l}^{\prime}\left(E_{0}\right)$ - момент полосы излучения порядка $l$ относительно $E_{0}$. При $T=0 E_{0}$ является общей точкой контуров обеих полос, при $T>0 \quad E_{0}$ есть точка пересечения контуров. Условие (13) означает требование равенства всех четных моментов, нечетные моменты должны отличаться только знаком. Впервые, насколько нам известно, это условие использовал для разбора проблемы зеркальной симметрии Кубарев $\left[{ }^{18}\right]$. Однако полученный им вывод о том, что зеркальная симметрия возможна вследствие некоторой статистической равноценности систем в среднем даже при отсутствии специальных требований, предъявляемых к адиабатическим потенциалам, прямо противоположен нашим результатам.

Согласно (5) моменты спектральных кривых имеют следующий вид:

$$
\begin{aligned}
& S_{i}^{*}\left(E_{0}\right)=\sum_{i}^{\top} n_{i} \sum_{s}\left(E_{s}^{\prime}-E_{i}-E_{0}\right)^{l} W_{s i}^{\mathrm{x}}, \\
& S_{l}^{\prime}\left(E_{0}\right)=\sum_{i} n_{i}^{\prime} \sum_{s}\left(E_{i}^{\prime}-E_{s}-E_{0}\right)^{l} W_{s i}^{\prime},
\end{aligned}
$$

где $n_{i}$ - функция распределения систем по колебательным уровням; $E_{i}$ - энергия колебательного уровня номера $i ; W_{s i}^{x_{i} I}-$ вероятность перехода с уровня $i$ на уровень $s$ при поглощении (индекс $x$ ) или при излучении (индекс I). Штрихованные величины относятся к возбужденному электронному состоянию.

Энергию электронно-колебательного перехода при поглощении можно выразить как сумму энергии чисто электронного перехода $\mathcal{E}$ и изменения колебательной энергии $\Delta E_{s i}^{x}$, а при излучении - как их разность: $\varepsilon-\Delta E_{s i}^{\prime}$.

Тогда выражения для моментов принимают вид

$$
\begin{aligned}
& S^{{ }^{*}}\left(E_{0}\right)=\sum_{i} n_{i} \sum_{s}\left(\Delta E_{s i}^{\times}+\mathcal{\varepsilon}-E_{0}\right)^{l} W_{s i}^{*}, \\
& S_{l}^{I}\left(E_{0}\right)=(-1)^{l} \sum_{i} n_{i}^{\prime} \sum_{s}\left(\Delta E_{s i}^{I}+E_{0}-\mathcal{E}\right)^{l} W^{l}{ }_{s_{i}}^{\prime}
\end{aligned}
$$

и соотношения (13) запишутся следующим образом:

$$
\begin{aligned}
& \sum_{i} n_{i} \sum_{s}\left(\Delta E_{s i}^{*}+\mathcal{E}-E_{0}\right)^{l} W_{s i}^{x}= \\
& =\sum_{i} n_{i}^{\prime} \sum_{s}\left(\Delta E_{s i}^{I}+E_{0}-\mathcal{E}\right)^{l} W_{s i}^{I} .
\end{aligned}
$$

Для того чтобы имели место соотношения (13a), дост аточно требовать выполнения следующих равенств: 


$$
\begin{gathered}
n_{i}=n_{i}^{\prime} \\
E_{s i}^{x}=\Delta E_{s i}^{I} \\
E_{0}=\mathcal{\varepsilon} \\
W_{s i}^{x}=W_{s i}^{I}
\end{gathered}
$$

Равенства (14) являются, по существу, условиями Левшина [ㄹ․

Второе из равенств (14) требует, чтобы спектры колебательных уровней были в обоих электронных состояниях одинаковы, а первое чтобы функции распределения систем по колебательным уровням были одинаковы в обоих электронных состояниях. В частности, если функции распределения по колебательным уровням термически равновесны, то они должны соответствовать одной и той же температуре в обоих электронных состояниях.

Третье равенство (14) означает, что «точкой симметрии» является энергия чисто электронного перехода. Покажем, что при выполнении остальных трех условий (14) контуры полос поглощения и излучения $J^{x}$ и $J^{I}$ пересекаются (при $T=0$ имеют общую точку) в точке $E=\mathcal{E}$. Действительно, в случае одинаковых спектров колебательных уровней в обоих электронных состояниях $J^{\star}(\mathcal{E})=\sum_{i} n_{i} W_{i i}^{x}$ и $J^{I}(\mathcal{E})=\sum_{i} n_{i}^{\prime} W_{i i}^{\prime}$, а в силу первого и последнего условий $(14) J^{x}(\mathcal{E})=J^{I}(\mathcal{E})$. Это значит, что при выполнении остальных трех условий (14) $\mathcal{E}$ является точкой пересечения контуров нормированных полос поглощения и излучения и тем самым $E_{0}=\mathcal{\ell}$. При весьма специальном выборе (неравновесных) распределений $n_{i}$ и $n_{i}^{\prime}$ могут быть и другие точки пересечения, но осуществление подобных случаев в действительности едва ли мыслимо. Кроме того, можно показать, что и тогда именно точка пересечения при $\mathcal{\varepsilon}$ является точкой симметрии $E_{0}$.

Последнее из равенств (14) запишется в приближении Кондона (электронный матричный элемент не зависит от ядерных координат). следующим образом:

$$
\left[\int \psi_{s}^{\prime}(x) \psi_{i}(x) d x\right]^{2}=\left[\int \psi_{s}(x) \psi_{i}^{\prime}(x) d x\right]^{2}
$$

где $\psi_{s}$ - колебательные волновые функции ядер. Выражение (15) можно рассматривать как равенство между квадратами коэффициентов разложения $\psi_{i}$ по $\psi_{s}^{\prime}$ и квадратами соответствующих коэффициентов разложения $\psi_{i}^{\prime}$ (того же номера $i$ ) по $\psi_{s}$. Это свидетельствует о существовании определенной симметрии волновых функций $\psi_{s}$ и $\psi_{s}^{\prime}$ друг относительно друга.

Условие одинаковости спектров колебательных уровней будет удовлетворено, если адиабатические потенциалы обоих электронных состояний имеют одинаковую форму, т. е. могут быть совмещены путем перемещения (параллельный перенос, отражение, инверсия и др. в многомерном пространстве $(x))$ одного из них без деформации в пространстве $(x)$ и сдвига вдоль оси энергии. Тогда и волновые функции $\psi_{s}$ и $\psi_{s}^{\prime}$ могут быть выбраны таким образом, что одну из них можно получить путем такого же перемещения другой в пространстве $(x)$, что привело к совпадению адиабатических потенциалов.

Однако выполнение последнего условия, относящегося к $\psi_{s}$ и $\psi_{s}^{\prime}$, еще отнюдь не всегда гарантирует выполнение равенства (15). Следует до- 
полнительно требовать, чтобы система из двух адиабатических потенциалов имела либо плоскость симметрии, либо центр симметрии в пространстве $(x)$. Например, когда один адиабатический потенциал можно получить путем параллельного переноса другого вдоль осей координат и оси энергии, для выполнения (15) надо, чтобы система из двух адиабатических потенциалов имела симметрию инверсии в пространстве $(x)$.

Проиллюстрируем условие существования центра или плоскости симметрии на примерах однокоординатных моделей.

А. Гармонический осциллятор неизменной частоты. Излучающей (или поглощающей) системой является гармонический осциллятор, частота колебания которого не изменяется в электронном переходе. Потенциальные кривые - параболы одинаковой крнвизны (фиг. 1a). Две параболы вместе образуют систему с центром симметрии в $x=0$. (Сдвиг вдоль оси энергии не следует принимать во внимание.) Из квантовомеханического расчета Пекара $\left.{ }^{11}\right]$ следует, что полосы поглощения и излучения такой модели действительно зеркально-симметричны.

Б. Ангармонический осциллятор. Для того чтобы потенциальные кривые ангармонического осциллятора образовали систему с центром симметрии, они должны обладать ангармоничностью различного знака для основного и возбужденного электронных состояний (кривые 1 и 2 на фиг. 16). Однако, положительный знак ангармоничности (кривая 2 на фиг. 16) в случае двухатомной молекулы или одной конфигурационной координаты весьма маловероятен. В реальном случае потенщиальных кривых с ангармоничностью одного знака (кривые 1 и 3 на фиг. 16) требуемой симметрин нет. Полосы поглощения и излучения, как это нетрудно увндеть из качественного рассмотрения соотношения (15),
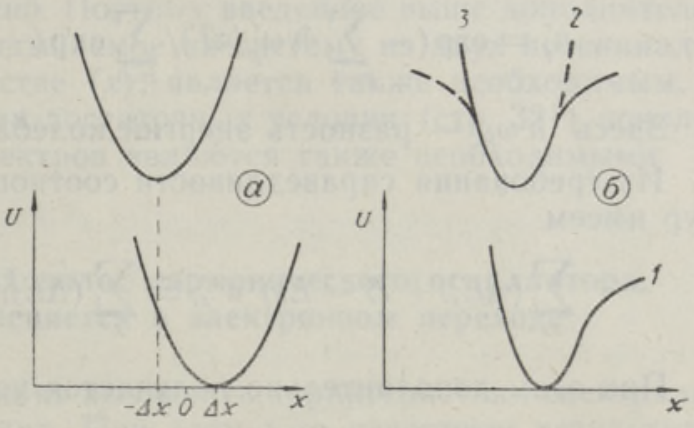

Фиг. 1. Диаграммы потенциальных кривых однокоординатных моделей центров люминесценции: $a$ - для гармонического осциллятора неизменной частоты, 6 - для ангармонического осциллятора. не обладают зеркальной симметрией, несмотря на то, что потенциальные кривые имеют одинаковую форму и спектры колебательных уровней одинаковы. Расчет конкретной модели осциллятора Морзе показал $\left[{ }^{20}\right]$, что в этом случае действительно возникают существенные отклонения от зеркальной симметрии контуров полос.

Из рассмотренных примеров видно, что п ракти чески условие существования центра или плоскости симметрин означает условие гармоничности колебаний и неизменности частот колебания в электронном переходе.

Итак, для появления зеркальной симметрии полос поглощения и излучения до с т а то чно:

во-первых, существования для системы из двух адиабатических потенциалов либо плоскости симметрии, либо центра симметрии в пространстве $(x)$;

во-вторых, одинаковости функций распределения систем по колебательным уровням в обоих электронных состояниях.

Выясним теперь, в какой мере перечисленные достаточны е условия являются также необходи мыми.

Прежде всего заметим, что при специальном выборе функций распределения $n_{i}$ и $n_{i}^{\prime}$ весьма часто возможно получение достаточно точной зеркальной симметрии при невыполнении условий (14). Однако ясно, что 
такие распределения практически невозможны. Также может случиться, что при термически равновесных распределениях при каких-то фиксированных температурах, различных для разных электронных состояний, соблюдается зеркальная симметрия, но при изменении температуры исчезает и симметрия спектров.

Поэтому мы в дальнейшем ограничимся случаем термически равновесных функций распределения по колебательным уровням $n_{i}$ и $n_{i}^{\prime}$, соответствующих олинаковым температурам. При этом мы будем понимать под зеркальной симметрией наличие ее для всех температур (практически для некоторого не слишком узкого интервала температур, начиная с температуры около $T=0$ ).

Итак, будем предполагать, что функции распределения систем по колебательным уровням $n_{i}$ и $n_{i}^{\prime}$ термически равновесны и соответствуют одной и той же температуре $T$.

$$
\begin{gathered}
n_{i}=\exp \left(-\sum_{p=1}^{i} \hbar \omega_{p} / \kappa T\right) / \sum_{i=0} \exp \left(-\sum_{p=1}^{i} \hbar \omega_{p} / \kappa T\right), \\
n_{i}^{\prime}=\exp \left(-\sum_{p=1}^{i} \hbar \omega_{p}^{\prime} / \kappa T\right) / \sum_{i=0} \exp \left(-\sum_{p=1}^{i} \hbar \omega_{p}^{\prime} / \kappa T\right) .
\end{gathered}
$$

Здесь $\hbar \omega_{p}$ - разность энергий колебательных уровней $p-1$ и $p$.

Из требования справедливости соотношений (13) для всех температур имеем

$$
\sum_{s}\left(\Delta E_{s i}^{x}+\varepsilon-E_{0}\right)^{l} W_{s i}^{x}=\sum_{s}\left(\Delta E_{s i}^{l}+E_{0}-\varepsilon\right)^{l} W_{s i}^{l} .
$$

При этом дополнительно получается условие

$$
\omega_{p}=\omega_{p}^{\prime} \text {, }
$$

т. е. спектры колебательных уровней в обоих электронных состояниях должны быть одинаковы. (18) означает, что $\Delta E_{s i}^{\star}=\Delta E_{s i}^{\prime}$. Из (16) и (18) следует, что одинаковы и функции распределения $n_{i}=n_{i}$.

В случае одинаковых спектров колебательных уровней в обоих электронных состояниях (условие (18)) контуры полос поглощения и нзлучения $I^{x}$ и $I^{I}$ пересекаются в точке $E=\mathscr{\varepsilon}$ независимо от температуры. Действительно, в силу (18) $J^{*}(\mathcal{E})=\sum_{i} n_{i} W_{i i}^{x}$ и $\quad J^{\prime}(\mathcal{E})=$ $=\sum_{i} n_{i} W_{i i}^{I}$, на основе $(16)$ и (18) $n_{i}=n_{i}^{\prime}$, а $W_{i i}^{x}=W_{i i}^{I}$. Отсюда $J^{\star}(\mathcal{E})=$ $=J^{I}(\boldsymbol{\varepsilon})$. Точка пересечения контуров полос является «точкой симметрии» полос: $E_{0}=\ell$.

Теперь можно (17) переписать в виде

$$
\sum_{s}\left(\Delta E_{s i}^{x}\right)^{l}\left(W_{s i}^{x}-W_{s i}^{I}\right)=0 .
$$

Левая часть этого равенства представляет собой момент порядка $l$ функции $W_{s i}^{x}-W_{s i}^{I}$ в шкале энергий. Функция, моменты всех порядков которой равны нулю, и сама равна нулю и

$$
W_{s i}^{z}=W_{s i}^{I} \text {. }
$$


Итак, при предположении, что функции распределения систем по колебательным уровням $n_{i}$ и $n_{i}^{\prime}$ термически равновесны и соответствуют одной и той же температуре $T$, условия (14), достаточные для появления зеркальной симметрии полос поглощения и излучения, являются также необходимыми.

Осталось еще выяснить, какие необходимые условия вытекают из (14) для адиабатических потенциалов. Очевидно, что системы колебательных уровней энергии могут быть тождественны только тогда, когда потенциальные поверхности имеют совершенно одинаковую форму в смысле возможности получения одной из них путем перемещения второй в пространстве $(x)$ и сдвига вдоль оси $E$. Волновые функции мы можем также выбрать так, чтобы функции, соответствующие эквивалентным наборам квантовых чисел, получились одна из другой путем перемещення в пространстве $(x)$. Однако этого еще недостаточно для того, чтобы соблюдалось также равенство вероятностей эквивалентных переходов (19). Если волновые функции в парах $\psi_{i}$ и $\psi_{s}^{\prime}, \psi_{i}^{\prime}$ и $\psi_{s}$ будут взаимно перекрываться своими неравноценными участками, то интегралы в обеих частях равенства (15), естественно, не равны между собой и условие (19) не будет удовлетворено. Поэтому введенное выше дополнительное условие симметрии, накладываемое на систему из двух потенциальных поверхностей в пространстве $(x)$, является также необходимым, и мы приходим к выводу, что два достаточных условия (стр. 321) появления зеркальной симметрии спектров являются также необходимыми.

\section{3. Моменты спектральных полос гармонического осциллятора, частота которого изменяется в электронном переходе}

На основе формулы (8) можно вычислить характеристики спектральных полос в различных случаях. При этом уже известные результаты получаются, как нам кажется, значительно более простым путем и в более удобном виде, чем другими методами. Кроме того, оказывается возможным получение ряда новых результатов. Изложение большинства из них является предметом отдельной статьи. В настоящем сообщении мы ограничимся выводом нескольких характеристик в простейших случаях, необходимых в связи с обсуждением общих свойств симметрии спектров. Не будем мы останавливаться и на расчетных подробностях.

Ограничимся приближением Кондона и рассмотрим сначала случай одного гармонического осциллятора. Выпишем формулы для полосы поглощения, а потом укажем простые правила получения из них формул для излучения. Явное выражение для распределения координаты гармонического осциллятора в тепловом равновесии хорошо известно [21]:

$$
\mathrm{Q}(x)=\frac{K}{\sqrt{\pi}} \exp \left(-K^{2} x^{2}\right),
$$

где $K=\sqrt{m \omega_{1}^{2} / 2 k \tau_{1}}$;

$m$ - масса осциллятора;

$\omega_{1}$ - частота осциллятора в основном электронном состоянии;

$\tau_{1}=\hbar \omega_{1} / 2 k t h\left(\hbar \omega_{1} / 2 k T_{1}\right)$;

$T_{1}$ - абсолютная температура в основном электронном состоянии.

Интегрирование выражений $(9 \mathrm{~B})-(9 д)$ при плотности $\varrho(x)$, взятой согласно (20), дает первые четыре начальных момента в виде: 


$$
\begin{gathered}
S_{0}=1 \text { (полоса нормирована на единицу) } \\
S_{1}=U^{\prime}(0)+\frac{k \tau_{1}}{2}\left(\frac{2 a^{\prime}}{m \omega_{1}^{2}}-1\right) \\
S_{2}=\left[U^{\prime}(0)\right]^{2}+k \tau_{1}\left[4 \frac{\left(a^{\prime} x_{0}^{\prime}\right)^{2}}{m \omega_{1}^{2}}+\left(\frac{2 a^{\prime}}{m \omega_{1}^{2}}-1\right) U^{\prime}(0)\right]+ \\
+3\left[\frac{k \tau_{1}}{2}\left(\frac{2 a^{\prime}}{m \omega_{1}^{2}}-1\right)\right]^{2} \\
+\frac{k \tau_{1}}{2}\left\{\hbar^{2} \omega_{1}^{2}\left(\frac{2 a^{\prime}}{m \omega_{1}^{2}}-1\right)^{2}+24 \frac{\left(a^{\prime} x_{0}^{\prime}\right)^{2}}{m \omega_{1}^{2}} U^{\prime}(0)+3\left(\frac{2 a^{\prime}}{m \omega_{1}^{2}}-1\right)\left[U^{\prime}(0)\right]^{2}\right\}+ \\
+\left(\frac{k \tau_{1}}{2}\right)^{2}\left\{9\left(\frac{2 a^{\prime}}{m \omega_{1}^{2}}-1\right)^{2}\left(U^{\prime} x_{0}^{\prime}\right)^{2}\right. \\
\left.S_{3}(0)+72 \frac{\left(a^{\prime} x_{0}^{\prime}\right)^{2}}{m \omega_{1}^{2}}\left(\frac{2 a^{\prime}}{m \omega_{1}^{2}}-1\right)\right\}+ \\
+15\left[\frac{k \tau_{1}}{2}\left(\frac{2 a^{\prime}}{m \omega_{1}^{2}}-1\right)\right]^{3} \cdot
\end{gathered}
$$

Здесь исползуются обозначения:

$U^{\prime}(0)$ - расстояние между адиабатическими потенциалами возбужденного и основного электронных состояний в минимуме потенциальной крнвой основного состояния;

$$
a^{\prime} \equiv \frac{1}{2}\left[\frac{d^{2} U_{I I}(x)}{d x^{2}}\right]_{x=0} ; \quad x_{0}^{\prime} \equiv-\frac{1}{2 a^{\prime}}\left[\frac{d U_{I I}(x)}{d x}\right]_{x=0},
$$

где $U_{\mathrm{II}}(x)$ - адиабатический потенциал возбужденного электронного состояния.

Для соответствующих центральных моментов получаем отсюда:

$$
\begin{aligned}
& \bar{S}_{0}=1 \\
& \bar{S}_{1}=0 \\
& \bar{S}_{2}=4 \frac{\left(a^{\prime} x_{0}^{\prime}\right)^{2}}{m \omega_{1}^{2}} k \tau_{1}+2\left[\frac{k \tau_{1}}{2}\left(\frac{2 a^{\prime}}{m \omega_{1}^{2}}-1\right)\right]^{2} \\
& \bar{S}_{3}=\frac{2 \hbar^{2}\left(a^{\prime} x_{0}^{\prime}\right)^{2}}{m}+\frac{k \tau_{1}}{2} \hbar^{2} \omega_{1}^{2}\left(\frac{2 a^{\prime}}{m \omega_{1}^{2}}-1\right)^{2}+ \\
& \quad+12\left(k \tau_{1}\right)^{2} \frac{\left(a^{\prime} x_{0}^{\prime}\right)^{2}}{m \omega_{1}^{2}}\left(\frac{2 a^{\prime}}{m \omega_{1}^{2}}-1\right)+\left[k \tau_{1}\left(\frac{2 a^{\prime}}{m \omega_{1}^{2}}-1\right)\right]^{3} .
\end{aligned}
$$

Если колебания в возбужденном электронном состоянии также считать точно гармоническими, то $a^{\prime}$ можно заменить на $a^{\prime}=m \omega_{2}^{2} / 2$, где $\omega_{2}$ - частота осциллятора в возбужденном электронном состоянии.

Формулы (21) и (22) записаны для полосы поглощения. Чтобы по- 
лучить формулы для излучения, следует моменты нечетного порядка умножить на $-1 ; \omega_{1}, T_{1}$ заменить на $\omega_{2}, T_{2}$ и $U^{\prime}(0), a^{\prime}, x_{0}^{\prime}$ на $U(0), a, x_{0}$, где $U(0)$ - расстояние между адиабатическими потенциалами основного и возбужденного электронных состояний в минимуме потенциальной кривой возбужденного состояния $(U(0)<0) ; T_{2}-$ абсолютная температура в возбужденном электронном состоянии, а $a$ и $x_{0}$ определены потенциалом $U_{\mathrm{I}}(x)$ и его производными в этом же минимуме.

Для системы независимых гармонических осцилляторов (рассмотрение колебательного, движения молекулы или кристалла в исходном электронном состоянии в приближении нормальных колебаний) интегралы распадутся на суммы интегралов и расчет дает начальные моменты в виде: *

$$
\begin{aligned}
& S_{0}=1 \\
& S_{1}=\sum_{j} S_{1 j} \\
& S_{2}=\sum_{j} S_{2 j}+\sum_{j \neq k}\left\{c _ { j } ^ { \prime } \left[c_{k}^{\prime}+k \tau_{k}\left(\frac{a_{k}^{\prime}}{a_{k}}-1\right)+\right.\right. \\
&\left.\quad+\frac{k \tau_{j}}{2} \frac{k \tau_{k}}{2}\left[\frac{1}{2} \frac{\left(a_{j k}^{\prime}\right)^{2}}{a_{j} a_{k}}+\left(\frac{a_{j}^{\prime}}{a_{j}}-1\right)\left(\frac{a_{k}^{\prime}}{a_{k}}-1\right)\right]\right\}
\end{aligned}
$$

$$
\begin{aligned}
S_{3}= & c^{3}+\frac{\hbar 2}{2} \sum_{j} \frac{\left(b_{j}^{\prime}\right)^{2}}{m_{j}}+\sum_{j} \frac{k \tau_{j}}{2}\left\{\frac{2{ }^{2} a_{j}}{m_{j}}\left(\frac{a_{j}^{\prime}}{a_{j}}-1\right)^{2}+3 c\left[\frac{\left(b_{j}^{\prime}\right)^{2}}{a_{j}}+c\left(\frac{a_{j}^{\prime}}{a_{j}}-1\right)\right]\right\}+ \\
& +9 \sum_{j}\left(\frac{k \tau_{j}}{2}\right)^{2}\left(\frac{a_{j}^{\prime}}{a_{j}}-1\right)\left[\frac{\left(b_{j}^{\prime}\right)^{2}}{a_{j}}+c\left(\frac{a_{j}^{\prime}}{a_{j}}-1\right)\right]+15 \sum_{j}\left(\frac{k \tau_{j}}{2}\right)^{3}\left(\frac{a_{j}^{\prime}}{a_{j}}-1\right)^{3}+ \\
+ & \sum_{j \neq k} \frac{k \tau_{j}}{2} \frac{\hbar^{2}}{2 m_{k}} \frac{\left(a_{j k}^{\prime}\right)^{2}}{a_{j}}+3 \sum_{j \neq k} \frac{k \tau_{j}}{2} \frac{k \tau_{k}}{2}\left\{\frac{1}{2} c \frac{\left(a_{j k}^{\prime}\right)^{2}}{a_{j} a_{k}}+\left(\frac{a_{k}^{\prime}}{a_{k}}-1\right)\left[\frac{\left(b_{j}^{\prime}\right)^{2}}{a_{j}}+c\left(\frac{a_{j}^{\prime}}{a_{j}}-1\right)\right]+\right.
\end{aligned}
$$$$
\left.+\frac{a_{j k}^{\prime} b_{j}^{\prime} b_{k}^{\prime}}{a_{j} a_{k}}\right\}+9 \sum_{j \neq k}\left(\frac{k \tau_{j}}{2}\right)^{2} \frac{k \tau_{k}}{2}\left(\frac{a_{j}^{\prime}}{a_{j}}-1\right)\left[\frac{\left(a_{j k}^{\prime}\right)^{2}}{a_{j} a_{k}}+\left(\frac{a_{j}^{\prime}}{a_{j}}-1\right)\left(\frac{a_{k}^{\prime}}{a_{k}}-1\right)\right]+
$$$$
+\sum_{j \neq k \neq l} \frac{k \tau_{j}}{2} \frac{k \tau_{k}}{2} \frac{k \tau_{l}}{2}\left[\frac{a_{j k}^{\prime} a_{j l}^{\prime} a_{k l}^{\prime}}{a_{j} a_{k} a_{l}}+\frac{3}{2} \frac{\left(a_{j k}^{\prime}\right)^{2}}{a_{j} a_{k}}\left(\frac{a_{l}^{\prime}}{a_{l}}-1\right)+\left(\frac{a_{j}^{\prime}}{a_{j}}-1\right)\left(\frac{a_{k}^{\prime}}{a_{k}}-1\right)\left(\frac{a_{l}^{\prime}}{a_{l}}-1\right)\right] .
$$

Здесь введены следующие обозначения: адиабатический потенциал основного электронного состояния $U_{I}=\sum_{j} a_{j} x_{j}^{2}$, а адиабатический потенциал возбужденного электронного состояния $U_{\mathrm{II}}=\frac{1}{2} \sum_{j \neq k} a_{i k}^{\prime} x_{j} x_{k}+$ $+\sum_{j}\left(a_{j}^{\prime} x_{j}^{2}+b_{j}^{\prime} x_{j}+c_{j}^{\prime}\right) ; c=\sum_{j} c_{j}^{\prime} ; \quad x_{j}-$ нормальные координаты; $S_{1 j}$ $S_{2 j}$ - начальные моменты в однокоординатном случае; $m_{j}-$ масса j-го осциллятора.

Соответствующие центральные моменты:

- Расчеты выполнены дипломантом ТГУ В. Хижняковым. Моменты $S_{1}, S_{2}$ были недавно вычислены также Абаренковым [ $\left.{ }^{22}\right]$. 


$$
\begin{gathered}
\bar{S}_{2}=\sum_{j} \bar{S}_{2 j}+\frac{1}{2} \sum_{j \neq k} \frac{k \tau_{j}}{2} \frac{k \tau_{k}}{2} \frac{\left(a_{j k}^{\prime}\right)^{2}}{a_{j} a_{k}} \\
S_{3}=\sum_{j} \bar{S}_{3 j}+\sum_{j \neq k}\left[\frac{k \tau_{j}}{2} \frac{\hbar^{2}}{2 m_{k}} \frac{\left(a_{j k}^{\prime}\right)^{2}}{a_{j}}+3 \frac{k \tau_{j}}{2} \frac{k \tau_{k}}{2} \frac{a_{j k}^{\prime} b_{j}^{\prime} b_{k}^{\prime}}{a_{j} a_{k}}+\right. \\
\left.+6\left(\frac{k \tau_{j}}{2}\right)^{2} \frac{k \tau_{k}}{2}\left(\frac{a_{j}^{\prime}}{a_{j}}-1\right) \frac{\left(a_{j k}^{\prime}\right)^{2}}{a_{j} a_{k}}\right]+\sum_{j \neq k \neq l} \frac{k \tau_{j}}{2} \frac{k \tau_{k}}{2} \frac{k \tau_{l}}{2} \frac{a_{j k}^{\prime} a_{j l}^{\prime} a_{k l}^{\prime}}{a_{j} a_{k} a_{l}},
\end{gathered}
$$

где $\bar{S}_{2 j}, \bar{S}_{3 j}$ - центральные моменты в однокоординатном случае.

\section{4. Связь между контурами полос поглощения и излучения} в случае изменения частот колебаний при электронном переходе

Из полученных в $\$ 3$ выражений для первых моментов видно, что в случае изменения частоты колебания зеркальной симметрии спектров нет, как это, согласно § 2 , и должно быть.

Формулы (21) вместе с правилами записи их для излучения устанавливают, по существу, связь между контурами полос. В целях сравнения с законом зеркальной симметрии Левшина представляет интерес записать моменты, взятые относительно электронной энергии $\mathcal{E}$. Ограничимся для простоты одним осциллятором. Из (21) получаем:

$$
\left.\begin{array}{l}
S_{0}^{\times}(\mathcal{E})=S_{0}^{I}(\mathcal{E})=1 \\
S_{1}^{\alpha}(\mathcal{E})=p^{\alpha}+\frac{k \tau}{2}\left(\frac{p^{x}}{p^{I}}-1\right) \\
S_{1}^{I}(\mathcal{C})=-p^{I}+\frac{k \tau^{\prime}}{2}\left(1-\frac{p^{I}}{p^{x}}\right)
\end{array}\right\}
$$

$$
\left.\begin{array}{l}
S_{2}^{\times}(\mathcal{E})=\left(p^{x}\right)^{2}+k \tau p^{x}\left(3 \frac{p^{x}}{p^{I}}-1\right)+3\left[\frac{k \tau}{2}\left(\frac{p^{x}}{p^{I}}-1\right)\right]^{2} \\
S_{2}^{I}(\mathcal{E})=\left(p^{I}\right)^{2}+k \tau^{\prime} p^{I}\left(3 \frac{p^{I}}{p^{x}}-1\right)+3\left[\frac{k \tau^{\prime}}{2}\left(1-\frac{p^{I}}{p^{x}}\right)\right]^{2}
\end{array}\right\}
$$

$$
\begin{aligned}
S_{3}^{\mathrm{x}}(\mathcal{E})= & \left(p^{\mathrm{x}}\right)^{3} \dashv-\hbar^{2} \omega_{2}^{2} p^{\mathrm{x}}+\frac{k \tau}{2}\left[\hbar^{2} \omega_{1}^{2}\left(\frac{p^{\mathrm{x}}}{p^{I}}-1\right)^{2}+3\left(p^{\mathrm{x}}\right)^{2}\left(5 \frac{p^{\mathrm{x}}}{p^{I}}-1\right)\right]+ \\
& +\left(\frac{k \tau}{2}\right)^{2} 9 p^{\mathrm{x}}\left(\frac{p^{\mathrm{x}}}{p^{I}}-1\right)\left(5 \frac{p^{\mathrm{x}}}{p^{I}}-1\right)+15\left[\frac{k \tau}{2}\left(\frac{p^{\mathrm{x}}}{p^{I}}-1\right)\right]^{3} \\
S_{3}^{I}(\mathcal{E})= & -\left(p^{I}\right)^{3}-\hbar^{2} \omega_{1}^{2} p^{I}+\frac{k \tau^{\prime}}{2}\left[-\hbar^{2} \omega_{2}^{2}\left(1-\frac{p^{I}}{p^{\mathrm{x}}}\right)^{2}-3\left(p^{I}\right)^{2}\left(5 \frac{p^{I}}{p^{\mathrm{x}}}-1\right)\right]+ \\
& +\left(\frac{k \tau^{\prime}}{2}\right)^{2} 9 p^{I}\left(1-\frac{p^{I}}{p^{\mathrm{x}}}\right)\left(5 \frac{p^{I}}{p^{\mathrm{x}}}-1\right)+15\left[\frac{k \tau^{\prime}}{2}\left(1-\frac{p^{I}}{p^{\mathrm{x}}}\right)\right]^{3}
\end{aligned}
$$

* В данном случае электронная энергия $\mathcal{\&}$ не совпадает с точкой пересечения нормированных на единицу спектральных полос. $\mathcal{E}$ равна минимальной энергии поглощения или максимальной энергии излучения при низких (значительно ниже дебаевской температуры $\Theta$ ) температурах, и отсюда можно ее в принципе измерить. Нетрудно убедиться в том, что в результате перехода к описанию распределения интенсивностей как непрерывной функции имеет место соотношение $\left.\frac{x(\mathcal{E})}{I(\mathcal{E})}\right|_{\text {при } \mathrm{T} \ll \Theta}=\frac{\omega_{1}}{\omega_{2}}$. 
Здесь $p^{x}, p^{I}$ - стоксовые потери при поглощении и излучении соответственно, а $\tau^{\prime}=\hbar \omega_{2} / 2 k t h\left(\hbar \omega_{2} / 2 k T^{\prime}\right)\left(T^{\prime}\right.$ - температура в возбужденном электронном состоянии. Вообще говоря, $T^{\prime} \neq T$ ).

Из формул (25) нетрудно увидеть следующее:

1. При $\omega_{1}=\omega_{2}$ имеем $p^{x}=p^{l}$, условие (13) выполняется и получается зеркальная симметрия.

2. Первое слагаемое во всех выражениях (25) больше остальных примерно в $p^{i} / k T$ раз. Поэтому для первых моментов $(l=0,1,2,3)$ имеем пр иближен но весьма простую связь:

$$
\left.\begin{array}{l}
S_{l}^{\times}(\mathcal{E}) \cong\left(p^{x}\right)^{l}+\frac{k \tau}{2} l\left(p^{x}\right)^{l-1}\left[(2 l-1)^{\left.\frac{p^{x}}{p^{l}}-1\right]}\right. \\
S_{l}^{l}(\mathcal{E}) \cong\left(-p^{I}\right)^{l}+(-1)^{l} \frac{k \tau^{\prime}}{2} l\left(p^{I}\right)^{l-1}\left[(2 l-1) \frac{p^{I}}{p^{x}}-1\right]
\end{array}\right\}
$$

или, ограничиваясь только первыми членами,

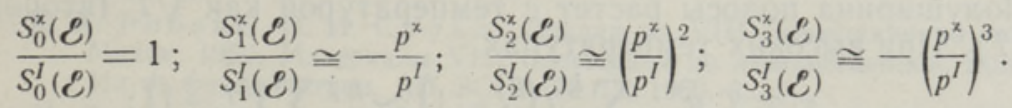

Следует подчеркнуть, что формулы (27) приближенны, причем приближение тем хуже, чем выше порядок момента. (Если (27) были точны, то это означало бы, что вместо полосы имеется одна единственная острая линия.) Однако практически требуемую информацию (при обычной точности экспериментального определения контуров полос) дают именно первые моменты. Кроме того, в реальных молекулах или кристаллофосфорах некоторое влияние на контуры оказывают также ангармоничность колебаний, отклонения от приближения Кондона, отклонения от адиабатического приближения и прочие более тонкие факторы. По крайней мере первые две из перечисленных причин способны изменить контур полосы в такой же мере, как члены, отбрасываемые при переходе к приближенным формулам (27).

Если считать, что система описывается нормальными колебаниями, у которых в результате электронного перехода изменяются лишь положения равновесия и частоты, но сами колебания не «перепутываются», то получим для первых моментов полосы поглощения, взятых относительно электронной энергии $\mathcal{E}$, нижеследующие формулы:

$$
\begin{aligned}
& S_{0}(\mathcal{E})=1 \\
& S_{1}(\mathcal{E})=p+\sum_{j} \frac{k \tau_{j}}{2}\left(\frac{p_{j}^{\prime}}{p_{j}}-1\right) \\
& S_{2}(\varepsilon)=\left[p+\sum_{j} \frac{k \tau_{j}}{2}\left(\frac{p_{j}^{\prime}}{p_{j}}-1\right)\right]^{2}+2 \sum_{j} k \tau_{j} p_{j}^{\prime} \bar{p}_{j}^{\prime}+ \\
&+2 \sum_{j}\left[\frac{k \tau_{j}}{2}\left(\frac{p_{j}^{\prime}}{p_{j}}-1\right)\right]^{2}
\end{aligned}
$$




$$
\begin{gathered}
S_{3}(\mathcal{L})=\left[p+\sum_{j} \frac{k \tau_{j}}{2}\left(\frac{p_{j}^{\prime}}{p_{j}}-1\right)\right]^{3}+6 p \sum_{j}\left\{k \tau_{j} p_{j}^{\prime} \frac{p_{j}^{\prime}}{p_{j}}+\left[\frac{k \tau_{j}}{2}\left(\frac{p_{j}^{\prime}}{p_{j}}-1\right)\right]^{2}\right\}+ \\
+6 \sum_{j} \frac{k \tau_{j}}{2}\left(\frac{p_{j}^{\prime}}{p_{j}}-1\right) \sum_{j} k \tau_{j} p_{j}^{\prime} \frac{p_{j}^{\prime}}{p_{j}}+6 \sum_{j} \frac{k \tau_{j}}{2}\left(\frac{p_{j}^{\prime}}{p_{j}}-1\right) \sum_{j}\left[\frac{k \tau_{j}}{2}\left(\frac{p_{j}^{\prime}}{p_{j}}-1\right)\right]^{2}+ \\
+\sum_{j}\left\{\hbar^{2} \omega_{j}^{\prime 2} p_{j}^{\prime}+\frac{k \tau_{j}}{2} \hbar^{2} \omega_{j}^{2}\left(\frac{p_{j}^{\prime}}{p_{j}}-1\right)^{2}+6\left(k \tau_{j}\right)^{2} p_{j}^{\prime} \frac{p_{j}^{\prime}}{p_{j}}\left(\frac{p_{j}^{\prime}}{p_{j}}-1\right)+8\left[\frac{k \tau_{j}}{2}\left(\frac{p_{j}^{\prime}}{p_{j}}-1\right)\right]^{3}\right\},
\end{gathered}
$$

тде $p=\sum_{j} p_{j}^{\prime}$

Чтобы получить формулы для излучения, следует моменты нечетного порядка умножить на -1 и $\tau_{j}, \omega_{j}, p_{j}, p_{j}^{\prime}$ заменить соответственно на $\tau_{j}^{\prime}, \omega_{j}^{\prime}, p_{j}^{\prime}$ и $p_{j}$.

Для центров люминесценции в кристаллофосфорах характерны большие стоксовые потери $(p \gg k T)$. Кроме того, сдвиг полосы (изменение первого момента) с изменением температуры является величиной порядка $k T$. Полуширина полосы растет с температурой как $\sqrt{T}$ (второй момент $S_{2} \sim T$ ). При высоких температурах

$$
\begin{aligned}
\tau_{j} \approx T \text { и } \quad & \sum_{j} \frac{k \tau_{j}}{2}\left(\frac{p_{j}^{\prime}}{p_{j}}-1\right) \approx \frac{k T}{2} \sum_{j}\left(\frac{p_{j}^{\prime}}{p_{j}}-1\right) ; \\
& \sum_{j} k \tau_{j} p_{j}^{\prime} p_{j}^{\prime} \approx k T p ; \\
& \sum_{j}\left[\frac{k \tau_{j}}{2}\left(\frac{p_{j}^{\prime}}{p_{j}}-1\right)\right]^{2} \approx\left(\frac{k T}{2}\right)^{2} \sum_{j}\left(\frac{p_{j}^{\prime}}{p_{j}}-1\right)^{2} .
\end{aligned}
$$

Поэтому в случае кристаллофосфоров из (28б) и (28в) вытекает, что суммы $\sum_{j}\left(\frac{p_{j}^{\prime}}{p_{j}}-1\right)$ и $\sum_{j}\left(\frac{p_{j}^{\prime}}{p_{j}}-1\right)^{2}$ не могут быть много больше еднницы. Следовательно, в многокоординатной модели можно при желании также ограничиваться первыми членами в (28) и прийти к весьма простым приближенным формулам связи (27).

\section{Л ИТ Е Р А У Р А}

1. M. I. a x, J. Chem. Phys., 20, 1952, 1752. (Перевод см. в сб. «Проблемы физики полупроводников», ИЛ, М., 1957.)

2. В. Л. Левшин, Фотолюминесценция жидких и твердых веществ, ГИТтЛ, М.-Л., 1951, стр. 96.

3. F. E. W i 11 i a m s, H. E y r ing, J. Chem. Phys., 15, 1947, 289; F. E. Willi a m s, M. H. H e b b, Phys. Rev., 84, 1951, 1181.

4. C. K 1 i c k, Phys. Rev., 85, 1952, 154.

5. F. E. Wil11 i m s, J. Chem. Phys., 19, 1951, 457; J. Phys. Chem., 57, 1953, 780.

6. Н. Е. Л ущик, Ч. Б. Л ущик, Тр. Ин-та физ. и астрон. АН ЭССР, № 6, 1957, 5; Ч. Б. Л ущик, Н. Е. Л ущик, К. К. Шв а ц, Тр. Ин-та физ. и астрон. АН ЭССР, № 8, 1958, 3. 
7. Н. Н. К ристофель, Тр. Ин-та физ. и астрон. АН ЭССР, № 10, 1959, 3; Тр. Ин-та физ. и астрон. АН ЭССР, № $7,1958,85 ;$ Н. Д. Потехина, Опт. и спектр., 2, 1957, 388.

8. К. К. Р еб ане, Тр. Ин-та физ. и астрон, АН ЭССР, № 7, 1958, 62.

9. Б. И. Степ анов, Люминесценция сложных молекул, ч. І, Изд. АН БССР, Минск, 1955.

10. Б. С. Н е п орен т, Тр. ГОИ, Сессия памяти С. И. Вавилова, Оборониздат, 1953.

11. С. И. Пек ар, Усп. физ. наук, 50, 1953, 197; С. И. Пека р, М. А. К ри вогл аз, Тр. Ин-та физ. АН УССР, вып. $4,1953,37$; А. Ф. Л уб ченк о, Опт. и спектр., 2, 1957, 195.

12. Huang Kun, A. Rhys, Proc. Roy. Soc. A 204, 1950, 406; R. Kubo, Y. To y oz a w a, Progr. Theor. Phys., 13, 1955, 160. (Перевод см. в сб. жПроблемы физики полупроводников», ИЛ, М., 1957.)

13. Б. В. Гн еден,к о, Курс теории вероятностей, М., 1954.

14. G. Russe 1 1, C. K 1 i c k, Phys. Rev., 101, 1956, 1473.

15. Л. П. К а заченко, Б. И. С теп ан ов, Опт. и спектр., 2, 1957, 339.

16. V. F o ck, Z. Phys., 89, 1934, 744; Е. Кондон, Г. Ш ортли, Теория атомных спектров, ИЛ, М., 1949, стр. 110.

17. Б. И. С те п а н в, Докл. АН СССР, 112, 1957, 839.

18. С. И. К у барев, Доклад на VIII Совещании по люминесценции, Минск, 1959; Материалы VIII Совещания по люминесценции, Минск, 1960.

19. В. М. Агранович, А. С. Давыдов, Уч. зап. Киевск. гос. ун-та, 14, вып. 8, $1955,15$.

20. К. К. Ребан е, О. И. С и льд, Доклад на VIII Совещании по люминесценции, Минск, 1959; Материалы VIII Совещания по люминесценции, Минск, 1960: Тр. Ин-та физ. и астрон. АН ЭССР, № 12, 1960, 3.

21. M. B or $\mathrm{n}$, Reports on Progress in Physics, 9, 1942-1943, 294, Appendix 1; H. O t t, Ann. Phys., 23, 1935, 169.

22. И. В. А баренко в, Диссертация на соискание ученой степени кандидата физико-математических наук, Ленингр. гос. ун-т, 1959.
Институт физики и астрономии
Академии наук Әстонской ССР
Поступила в редакцию
19. III 1960

\title{
MOLEKULIDE JA KRISTALLIDE ELEKTRON-VONKUMISSPEKTRITE TEOORIAST
}

\author{
K. Rebane,
}

\author{
füüsikalis-matemaatiliste teaduste kandidaat
}

\section{Sild}

\section{Resümee}

Käesolevas töös kirjeldatakse molekulide ja kristallide elektron-vōnkumisspektreid momentide meetodil, mida on esmakordselt kasutanud Lax [ $\left.{ }^{1}\right]$. Lihtsa tuletuskäigu abil on saadud spektraalriba momentide valem, mis ühtib Lax'i tulemusega. Valem on saadud üldistel eeldustel: võnkumiste kirjeldamisel ei kasutata normaalkoordinaate ja süsteemide jaotus võnkenivoodel ei pea olema tasakaaluline. Spektraalriba võib olla sealjuures osaliselt vōi tervenisti diskreetse struktuuriga. Momentide valemi tuletamisel pole nõutav adiabaatiline lähendus.

Antud momentide meetodi abil leitakse piisavad ja tarvilikud tingimused adiabaatiliste potentsiaalide ja süsteemide jaotuse kohta vōnkenivoodel neeldumis- ja kiirgusriba peegelsümmeetria tekkimiseks ning arvutatakse ühe harmoonilise ostsillaatori ja sõltumatute harmooniliste ostsillaatorite süsteemi spektraalribade momendid; ühtlasi näidatakse seos neeldumis- ja kiirgusriba vahel nende süsteemide jaoks. 


\title{
ON THE THEORY OF ELECTRON-VIBRATIONAL SPECTRA OF MOLECULES AND CRYSTALS
}

\author{
K. Rebane, O. Sild
}

\section{Summary}

The authors describe their investigations of the electron-vibrational spectra, in which they applied the method of moments that was used by Lax[1]. By simple derivation they obtained a formula for computing the moments of the spectral band. The formula is applicable within wide limits: normal coordinates are not used in describing the vibrations, and the distribution of systems over the vibrational levels need not be in thermal equilibrium. Further, there is no need for adiabatic approximation in deriving the formula for the moments.

This method was used to determine the necessary and sufficient conditions for the occurrence of mirror symmetry between the absorption and emission bands. The first four moments of the spectral bands were calculated for a system of independent harmonic oscillators (normal vibrations) whose equilibrium positions and vibration frequencies are both affected by electronic transition. The connection between the absorption and emission bands of those systems is established.

Academy of Sciences of the Estonian S.S.R., Received Institute of Physics and Astronomy 